\title{
Iterative Multiuser Minimum Symbol Error Rate Beamforming Aided QAM Receiver
}

\author{
Shuang Tan, Sheng Chen, and Lajos Hanzo
}

\begin{abstract}
A novel iterative soft interference cancellation (SIC) aided beamforming receiver is developed for high-throughput quadrature amplitude modulation systems. The proposed SICbased minimum symbol error rate (MSER) multiuser detection scheme guarantees the direct and explicit minimization of the symbol error rate at the output of the detector. Adopting the extrinsic information transfer (EXIT) chart technique, we compare the EXIT characteristics of an iterative MSER multiuser detector (MUD) with those of the conventional minimum mean-squared error (MMSE) detector. As expected, the proposed SIC-MSER MUD outperforms the SIC-MMSE MUD.
\end{abstract}

Index Terms-Beamforming, iterative multiuser detection, minimum symbol error rate, quadrature amplitude modulation.

\section{INTRODUCTION}

I TERATIVE detection principle, originally proposed in the context of turbo codes [1], [2], has found its way into iterative detector designs [3]-[6]. Most studies consider the minimum mean-square error (MMSE) soft interference cancellation (SIC) aided iterative multiuser detector (MUD) [4]-[6]. However, the MMSE algorithm does not guarantee the minimization of the system's error rate. In [7] and [8], the bit error rate (BER), rather than the mean-square error, was minimized at the MUD's output for binary phase shift keying and quadrature phase shift keying signals. Yeh and Barry [9] have succeeded in directly minimizing the detector's symbol error rate (SER) for quadrature amplitude modulation (QAM) signals. Recently, a minimum SER (MSER) beamforming aided receiver has been developed for QAM systems [10]. The concept of extrinsic information transfer (EXIT) charts was introduced in [11]. This semi-analytic technique uses the mutual information (MI) between the inputs and outputs of the concatenated receiver components in order to analyze their achievable performance. EXIT charts were employed in turbo equalization in [5], while in [12], they were used for examining the convergence properties of a turbo MUD. The novelty of this contribution is that iterative SIC aided MSER beamforming is proposed for QAM signals, and its performance is studied with the aid of EXIT charts.

\section{SYSTEM DESCRIPTION}

The system supports $K$ number of QAM users, and each user transmits on the same angular carrier frequency $\omega$. The receiver is equipped with a linear antenna array consisting of $L$ elements, which has a uniform element spacing of half wavelength. As-

Manuscript received October 1, 2007; revised December 17, 2007. The associate editor coordinating the review of this manuscript and approving it for publication was Prof. Richard J. Kozick.

The authors are with the School of Electronics and Computer Science, University of Southampton, Southampton SO17 1BJ, U.K.

Digital Object Identifier 10.1109/LSP.2008.917792

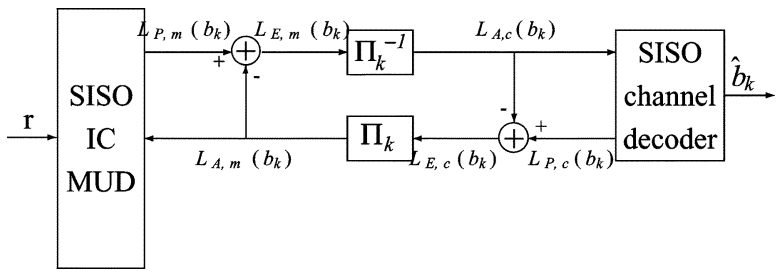

Fig. 1. Iterative multiuser beamforming receiver structure.

sume that the channel is non-dispersive. Then the symbol-ratesampled received signal can be expressed as $r_{l}(n)=$ $\sum_{k=1}^{K} h_{k} s_{k}(n) e^{j \omega t_{l}\left(\theta_{k}\right)}+n_{l}(n)$ for $1 \leq l \leq L$, where $h_{k}$ is the channel coefficient of user $k, s_{k}(n)$ is the $n$th symbol of the $k$ th user, $n_{l}(n)$ is a complex-valued additive white Gaussian noise process with $E\left[\left|n_{l}(n)\right|^{2}\right]=2 \sigma_{n}^{2}$, and $t_{l}\left(\theta_{k}\right)$ is the relative time delay at array element $l$ for the user $k$ signal, with $\theta_{k}$ being the direction of arrival for user $k$. The received signal vector $\mathbf{r}(n)=\left[r_{1}(n) r_{2}(n) \ldots r_{L}(n)\right]^{T}$ is given by $\mathbf{r}(n)=$ $\mathbf{H s}(n)+\mathbf{n}(n)$, where $\mathbf{n}(n)=\left[n_{1}(n) n_{2}(n) \ldots n_{L}(n)\right]^{T}$, $\mathbf{s}(n)=\left[s_{1}(n) s_{2}(n) \ldots s_{K}(n)\right]^{T}$, and the system matrix $\mathbf{H}=\left[\mathbf{h}_{1} \mathbf{h}_{2} \ldots \mathbf{h}_{K}\right]$, with the steering vectors $\mathbf{h}_{k}=$ $\left[h_{k} e^{j \omega t_{1}\left(\theta_{k}\right)} h_{k} e^{j \omega t_{2}\left(\theta_{k}\right)} \cdots h_{k} e^{j \omega t_{L}\left(\theta_{k}\right)}\right]^{T}, 1 \leq k \leq K$.

The iterative multiuser beamforming receiver's structure is shown in Fig. 1, which consists of two stages, namely, the soft-input soft-output (SISO) interference cancellation aided beamforming MUD, followed by $K$ parallel single-user SISO channel decoders. The two stages are separated by the usual bit-based deinterleavers $\Pi^{-1}$ and interleavers $\Pi$. The proposed SISO beamforming MUD first determines the beamformer weight vector $\mathrm{w}_{k}(n)$ according to the specific design criterion and provides an estimated symbol $\hat{s}_{k}(n)$ corresponding to the transmitted symbol $s_{k}(n)$ with the aid of linear transformation $\mathbf{w}_{k}^{H}(n) \mathbf{r}(n)$. Let us define $b_{k}(n, i)$ as the $i$ th $\left(i \in\left\{1, \ldots, \log _{2} M\right\}\right)$ bit of the $M$-QAM symbol $s_{k}(n)$, whereas $b_{k}(j)$ is the same bit but in a different position of the bit-based interleaving block after the deinterleaver. $L_{A}(\cdot)$, $L_{P}(\cdot)$, and $L_{E}(\cdot)$ denote the a priori, a posteriori, and extrinsic information in terms of logarithmic likelihood ratio (LLR), and the subscripts $m$ and $c$ are associated with the MUD and channel decoder, respectively. Then the SISO beamforming MUD delivers the a posteriori information of bit $b_{k}(n, i)$ expressed in terms of its LLR as [6]

$$
\begin{aligned}
L_{P, m}\left(b_{k}(n, i)\right) & =\ln \frac{\mathrm{P}\left[\hat{s}_{k}(n) \mid b_{k}(n, i)=0\right]}{\mathrm{P}\left[\hat{s}_{k}(n) \mid b_{k}(n, i)=1\right]}+\ln \frac{\mathrm{P}\left[b_{k}(n, i)=0\right]}{\mathrm{P}\left[b_{k}(n, i)=1\right]} \\
& =L_{E, m}\left(b_{k}(n, i)\right)+L_{A, m}\left(b_{k}(n, i)\right)
\end{aligned}
$$

where $L_{A, m}\left(b_{k}(n, i)\right)$ represents the a priori LLR of the interleaved and encoded bits $b_{k}(n, i)$, while $L_{E, m}\left(b_{k}(n, i)\right)$ represents the extrinsic information delivered by the SISO MUD, 


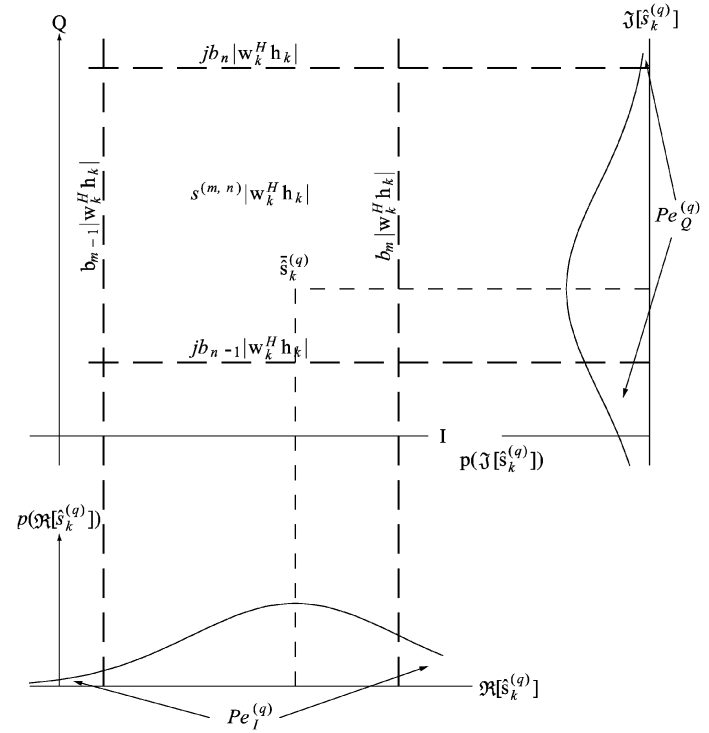

Fig. 2. Interference-affected expectation $\overline{\hat{s}}_{k}^{(q)}$ of the estimated signal $\hat{s}_{k}^{(q)}$ when $s_{k}^{(q)}=s^{(m, n)}$ and the decision boundaries.

based on the received signal $\mathbf{r}(n)$ and the a priori information about the encoded bits of all users, except for the $i$ th bit of the desired user $k$. The extrinsic information is then deinterleaved and fed into the $k$ th user's channel decoder, as the a priori information in the next iteration.

As seen in Fig. 1, between the banks of channel decoders and interleavers, we compute the extrinsic LLR based on the a priori information $L_{A, c}\left(b_{k}(j)\right)$ provided by the SISO MUD for the SISO decoder as $L_{E, c}\left(b_{k}(j)\right)=L_{P, c}\left(b_{k}(j)\right)-L_{A, c}\left(b_{k}(j)\right)[6]$, where the extrinsic information is gleaned from the surrounding encoded bits, excluding the specific bit considered [6]. After interleaving, the extrinsic information delivered by the channel decoders is fed back to the SISO MUD, as the a priori information concerning the encoded bits of all the users for exploitation during the next iteration.

\section{SISO INTERFERENCE CANCELLATION}

Given the a priori LLRs, the mean and variance based on the $a$ priori information of the $k$ th user's symbols are defined as [5]: $\bar{s}_{k}=\mathrm{E}\left[s_{k}\right]$ and $v_{k}=\mathrm{E}\left[\left|s_{k}\right|^{2}\right]-\left|\bar{s}_{k}\right|^{2}$, where the symbol-index $n$ was dropped for notational convenience. When using the SIC principle, the estimated symbol of user $k$ can be expressed as [5]: $\hat{s}_{k}=\mathbf{w}_{k}^{H}\left(\mathbf{r}-\mathbf{H} \underline{\mathbf{s}}_{k}\right)$, where $\underline{\mathbf{s}}_{k}=\left[\bar{s}_{1} \ldots \bar{s}_{k-1} 0 \bar{s}_{k+1} \ldots \bar{s}_{K}\right]^{T}$.

\section{A. SISO Interference Cancellation Using the MMSE MUD}

Classically, the MMSE solution for the beamformer's weight vector $\mathbf{w}_{k}$ is expressed as [6]

$$
\mathbf{w}_{k, m m s e}=\left(\mathbf{H} \underline{\mathbf{V}}_{k} \mathbf{H}^{H}+E_{s} \mathbf{h}_{k} \mathbf{h}_{k}^{H}+2 \sigma_{n}^{2} \mathbf{I}_{L}\right)^{-1} \cdot E_{s} \mathbf{h}_{k}
$$

where $E_{s}$ is the average symbol energy, $\mathbf{I}_{L}$ denotes the $L \times L$ identity matrix, and $\underline{\mathbf{V}}_{k}=\operatorname{diag}\left[v_{1} \ldots v_{k-1} 0 v_{k+1} \ldots v_{K}\right]$, in which $\operatorname{diag}[\cdot]$ denotes a diagonal matrix. The conditional probability density function (PDF) $\mathrm{P}\left[\hat{s}_{k} \mid s_{k}=s^{(p)}\right]$, where $s^{(p)}$ is the $p$ th $(p \in\{1,2, \ldots, M\})$ legitimate value of the QAM constellation, may be assumed to be Gaussian distributed and the corresponding extrinsic output LLR is given by [6]

$$
\begin{aligned}
L_{E}\left(b_{k}(i)\right)= & \sum_{\substack{\forall s^{(p)}: \\
b^{(p)}(i)=0}} \exp \left(\frac{-\left|\hat{s}_{k}-\mu_{k}^{(p)}\right|^{2}}{\sigma_{k}^{2}}\right) \prod_{\forall i^{\prime} \neq i} \mathrm{P}\left(b_{k}^{(p)}\left(i^{\prime}\right)\right) \\
\left.\ln \frac{-\left|\hat{s}_{k}-\mu_{k}^{(p)}\right|^{2}}{\sigma_{k}^{2}}\right) \prod_{\forall i^{\prime} \neq i} \mathrm{P}\left(b_{k}^{(p)}\left(i^{\prime}\right)\right) & \exp \left(\frac{\sum^{(p)}:}{b^{(p)}(i)=1}\right.
\end{aligned}
$$

where $b^{(p)}(i)$ denotes the $i$ th bit of $s^{(p)}, \mu_{k}^{(p)}=s^{(p)} \mathbf{w}_{k}^{H} \mathbf{h}_{k}$, $\sigma_{k}^{2}=E_{s} \mathbf{w}_{k}^{H} \mathbf{h}_{k}\left(1-\mathbf{w}_{k}^{H} \mathbf{h}_{k}\right)$, and the a priori probability of the $i^{\prime}$ th bit in symbol $s_{k}$ is $\mathrm{P}\left(b_{k}^{(p)}\left(i^{\prime}\right)\right)=(1 / 2)(1+\operatorname{sgn}((1 / 2)-$ $\left.\left.b^{(p)}\left(i^{\prime}\right)\right) \cdot \tanh \left(L_{A}\left(b_{k}\left(i^{\prime}\right)\right) / 2\right)\right)$.

\section{B. SISO Interference Cancellation Using the MSER MUD}

In [10], the MSER algorithm is investigated when the MUD has access to no a priori information. Under this condition, the subset PDFs conditioned on the different values of the estimated $\hat{s}_{k}$ satisfy the shifting properties and are symmetrically distributed [10]. However, when the MUD is provided with $a$ priori information, these properties are invalid and the MSER method of [10] cannot be applied directly to our iterative system. Hence, we derive a new a priori information aided MSER MUD. Define a symbol in the $M$-QAM constellation as $s^{(m, n)}=\gamma(2 m-\sqrt{M}-1)+j \cdot \gamma(2 n-\sqrt{M}-1)$, where $\gamma=\sqrt{3 E_{s}} / \sqrt{2(M-1)}$ and $1 \leq m, n \leq \sqrt{M}$. Assume that the $q$ th $\left(q \in\left\{1, \ldots, M^{K}\right\}\right)$ symbol combination $\mathbf{s}^{(q)}$ is transmitted, in which the desired user $k$ transmits symbol $s_{k}^{(q)}=s^{(m, n)}$. Fig. 2 shows the estimated $\hat{s}_{k}^{(q)}$ and its marginal PDFs. The PDF of $\hat{s}_{k}^{(q)}$ is Gaussian distributed with a mean $\overline{\hat{s}}_{k}^{(q)}=\mathbf{w}_{k}^{H}\left(\mathbf{H} \mathbf{s}^{(q)}-\mathbf{H} \underline{\mathbf{s}}_{k}\right)$, as seen in Fig. 2. When the $k$ th user transmits symbol $s^{(m, n)}$, the conditional PDF of $\hat{s}_{k}$ is a Gaussian mixture defined by

$$
\begin{gathered}
p\left(\hat{s}_{k} \mid s_{k}=s^{(m, n)}\right)=M \sum_{\substack{\forall \mathbf{s}^{(q)}: \\
s_{k}^{(q)}=s^{(m, n)}}} \underline{P}_{k}\left(\mathbf{s}^{(q)}\right) \cdot p\left(\hat{s}_{k}^{(q)} \mid s_{k}^{(q)}=s^{(m, n)}\right) \\
=\frac{M}{2 \pi \sigma_{n}^{2} \mathbf{w}_{k}^{H} \mathbf{w}_{k}} \sum_{\substack{\forall \mathbf{s}^{(q)}: \\
s_{k}^{(q)}=s^{(m, n)}}} \underline{P}_{k}\left(\mathbf{s}^{(q)}\right) \cdot \exp \left(-\frac{\left|\hat{s}_{k}-\bar{s}_{k}^{(q)}\right|^{2}}{2 \sigma_{n}^{2} \mathbf{w}_{k}^{H} \mathbf{w}_{k}}\right)
\end{gathered}
$$

where $\underline{P}_{k}\left(\mathbf{s}^{(q)}\right)=1 / M \prod_{\forall k^{\prime} \neq k} \mathrm{P}\left(s_{k^{\prime}}=s_{k^{\prime}}^{(q)}\right)$ is the probability of transmitting the $q$ th possible symbol combination $\mathbf{s}^{(q)}$, given the a priori information of the other $(K-1)$ users, except for user $k$. By defining $b_{i}=\gamma(2 i-\sqrt{M})$ for $1 \leq i \leq \sqrt{M}-1$, the decision boundaries of $\hat{s}_{k}$ are determined by $b_{i}\left|\mathbf{w}_{k}^{H} \mathbf{h}_{k}\right|$ for the in-phase component and by $j b_{i}\left|\mathbf{w}_{k}^{H} \mathbf{h}_{k}\right|$ for the quadrature component, as seen in Fig. 2. Fig. 2 only portrays the scenario of the inner constellation point, which is enclosed by boundaries. The points at the edge of the constellation may have open boundaries in one or two directions. 
Assume that the $k$ th user transmits symbol $s^{(m, n)}$. The in-phase component's conditional error probability of $\Re\left[\hat{s}_{k}\right] \neq \Re\left[s^{(m, n)}\right]$ can be shown to be (also see [10])

$$
\begin{aligned}
& P e_{I}\left(s_{k}=s^{(m, n)}\right) \\
& \int \sum_{\substack{\forall \mathbf{s}^{(q)}: \\
s_{k}^{(q)}=s^{(m, n)}}} \underline{P}_{k}\left(\mathbf{s}^{(q)}\right) \cdot \mathrm{Q}\left(\frac{b_{1}\left|\mathbf{w}_{k}^{H} \mathbf{h}_{k}\right|-\Re\left[\overline{\hat{s}}_{k}^{(q)}\right]}{\sigma_{n} \sqrt{\mathbf{w}_{k}^{H} \mathbf{w}_{k}}}\right), m=1
\end{aligned}
$$

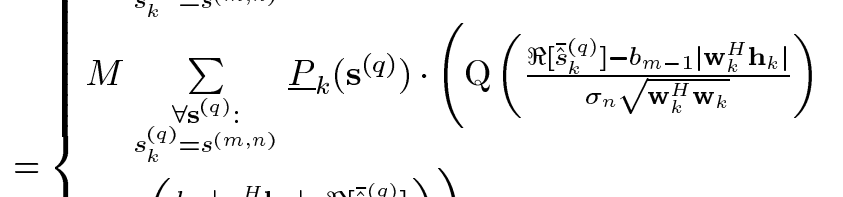

$$
\begin{aligned}
& \left.+\mathrm{Q}\left(\frac{b_{m}\left|\mathbf{w}_{k}^{H} \mathbf{h}_{k}\right|-\Re\left[\overline{\hat{s}}_{k}^{(q)}\right]}{\sigma_{n} \sqrt{\mathbf{w}_{k}^{H} \mathbf{w}_{k}}}\right)\right), 2 \leq m \leq \sqrt{M}-1 \\
& M \sum_{\forall \mathbf{s}^{(q)}:} \quad \underline{P}_{k}\left(\mathbf{s}^{(q)}\right) \cdot \mathrm{Q}\left(\frac{\Re\left[\overline{\hat{s}}_{k}^{(q)}\right]-b_{\sqrt{M}-1}\left|\mathbf{w}_{k}^{H} \mathbf{h}_{k}\right|}{\sigma_{n} \sqrt{\mathbf{w}_{k}^{H} \mathbf{w}_{k}}}\right) \\
& s_{k}^{(q)}=s^{(m, n)} \\
& m=\sqrt{M}
\end{aligned}
$$

Similarly, the quadrature component's conditional error probability of $\Im\left[\hat{s}_{k}\right] \neq \Im\left[s^{(m, n)}\right]$ can be shown to be

$$
\begin{aligned}
& P e_{Q}\left(s_{k}=s^{(m, n)}\right) \\
& =\left\{\begin{array}{c}
M \sum_{\substack{\forall \mathbf{s}^{(q)}: \\
s_{k}^{(q)}=s^{(m, n)}}} \underline{P}_{k}\left(\mathbf{s}^{(q)}\right) \cdot \mathrm{Q}\left(\frac{b_{1}\left|\mathbf{w}_{k}^{H} \mathbf{h}_{k}\right|-\Im\left[\overline{\hat{s}}_{k}^{(q)}\right]}{\sigma_{n} \sqrt{\mathbf{w}_{k}^{H} \mathbf{w}_{k}}}\right), n=1 \\
M \sum_{\substack{\forall \mathbf{s}^{(q)}: \\
s_{k}^{(q)}=s^{(m, n)}}} \underline{P}_{k}\left(\mathbf{s}^{(q)}\right) \cdot\left(\mathrm{Q}\left(\frac{\Im\left[\overline{\hat{s}}_{k}^{(q)}\right]-b_{n-1}\left|\mathbf{w}_{k}^{H} \mathbf{h}_{k}\right|}{\sigma_{n} \sqrt{\mathbf{w}_{k}^{H} \mathbf{w}_{k}}}\right)\right. \\
\left.+\mathrm{Q}\left(\frac{b_{n}\left|\mathbf{w}_{k}^{H} \mathbf{h}_{k}\right|-\Im\left[\overline{\hat{s}}_{k}^{(q)}\right]}{\sigma_{n} \sqrt{\mathbf{w}_{k}^{H} \mathbf{w}_{k}}}\right)\right) \\
\sum_{\substack{\forall \mathbf{s}^{(q)}: \\
s_{k}^{(q)}=s^{(m, n)}}} \underline{P}_{k}\left(\mathbf{s}^{(q)}\right) \cdot \mathrm{Q}\left(\frac{\Im\left[\hat{s}_{k}^{(q)}\right]-b \sqrt{M}-1}{\sigma_{n} \sqrt{\mathbf{w}_{k}^{H} \mathbf{w}_{k}}\left|\mathbf{w}_{k}^{H} \mathbf{h}_{k}\right|}\right) \\
n=\sqrt{M}
\end{array}\right.
\end{aligned}
$$

The average error probabilities of the in-phase and quadrature components are given, respectively, by

$$
\begin{aligned}
P e_{I} & =\frac{1}{M} \sum_{m=1}^{\sqrt{M}} \sum_{n=1}^{\sqrt{M}} P e_{I}\left(s_{k}=s^{(m, n)}\right) \\
P e_{Q} & =\frac{1}{M} \sum_{m=1}^{\sqrt{M}} \sum_{n=1}^{\sqrt{M}} P e_{Q}\left(s_{k}=s^{(m, n)}\right) .
\end{aligned}
$$

The resultant SER can be formulated as $P e_{s}=P e_{I}+P e_{Q}-$ $P e_{I} \cdot P e_{Q}$, and the MSER solution is defined as the one that minimizes the upper bound of the SER given by

$$
\mathbf{w}_{k, m s e r}=\arg \min _{\mathbf{w}_{k}}\left(P e_{I}+P e_{Q}\right)
$$

In order to arrive at an optimum MSER solution, we need the gradients of $P e_{I}$ and $P e_{Q}$ in the context of the simplified con- jugate gradient algorithm [7], which can be derived from the gradients of the Q-functions in (5) and (6), leading to

$\nabla_{\mathbf{w}_{k}} \mathrm{Q}\left(\frac{\Re\left[\overline{\hat{s}}_{k}^{(q)}\right]-b_{i}\left|\mathbf{w}_{k}^{H} \mathbf{h}_{k}\right|}{\sigma_{n} \sqrt{\mathbf{w}_{k}^{H} \mathbf{w}_{k}}}\right)=$

$\frac{1}{\sqrt{2 \pi} \sigma_{n} \sqrt{\mathbf{w}_{k}^{H} \mathbf{w}_{k}}} \cdot \exp \left(-\frac{\left(\Re\left[\overline{\hat{s}}_{k}^{(q)}\right]-b_{i}\left|\mathbf{w}_{k}^{H} \mathbf{h}_{k}\right|\right)^{2}}{2 \sigma_{n}^{2} \mathbf{w}_{k}^{H} \mathbf{w}_{k}}\right)$
$\cdot\left(\frac{\mathbf{w}_{k}\left(\Re\left[\overline{\hat{s}}_{k}^{(q)}\right]-b_{i}\left|\mathbf{w}_{k}^{H} \mathbf{h}_{k}\right|\right)}{\mathbf{w}_{k}^{H} \mathbf{w}_{k}}-\overline{\mathbf{r}}_{k}^{(q)}+\frac{b_{i} \mathbf{h}_{k} \mathbf{h}_{k}^{H} \mathbf{w}_{k}}{\left|\mathbf{w}_{k}^{H} \mathbf{h}_{k}\right|}\right)$

and

$\nabla_{\mathbf{w}_{k}} \mathrm{Q}\left(\frac{\Im\left[\hat{s}_{k}^{(q)}\right]-b_{i}\left|\mathbf{w}_{k}^{H} \mathbf{h}_{k}\right|}{\sigma_{n} \sqrt{\mathbf{w}_{k}^{H} \mathbf{w}_{k}}}\right)=$

$\frac{1}{\sqrt{2 \pi} \sigma_{n} \sqrt{\mathbf{w}_{k}^{H} \mathbf{w}_{k}}} \cdot \exp \left(-\frac{\left(\Im\left[\overline{\hat{s}}_{k}^{(q)}\right]-b_{i}\left|\mathbf{w}_{k}^{H} \mathbf{h}_{k}\right|\right)^{2}}{2 \sigma_{n}^{2} \mathbf{w}_{k}^{H} \mathbf{w}_{k}}\right)$

$\cdot\left(\frac{\mathbf{w}_{k}\left(\Im\left[\bar{s}_{k}^{(q)}\right]-b_{i}\left|\mathbf{w}_{k}^{H} \mathbf{h}_{k}\right|\right)}{\mathbf{w}_{k}^{H} \mathbf{w}_{k}}+j \overline{\mathbf{r}}_{k}^{(q)}+\frac{b_{i} \mathbf{h}_{k} \mathbf{h}_{k}^{H} \mathbf{w}_{k}}{\left|\mathbf{w}_{k}^{H} \mathbf{h}_{k}\right|}\right)$

where $\overline{\mathbf{r}}_{k}^{(q)}=\mathbf{H} \mathbf{s}^{(q)}-\mathbf{H} \underline{\mathbf{s}}_{k}$.

The marginal conditional PDFs $p\left(\Re\left[\hat{s}_{k}\right] \mid s_{k}=s^{(m, n)}\right)$ and $p\left(\Im\left[\hat{s}_{k}\right] \mid s_{k}=s^{(m, n)}\right)$ can both be assumed to be Gaussian distributed. The means and variances of the in-phase and quadrature components of $\hat{s}_{k}$ are given by $\mu_{k, I}^{(m, n)}=\Re\left[s^{(m, n)} \mathbf{w}_{k}^{H} \mathbf{h}_{k}\right]$, $\mu_{k, Q}^{(m, n)}=\Im\left[s^{(m, n)} \mathbf{w}_{k}^{H} \mathbf{h}_{k}\right]$, and

$$
\begin{aligned}
\sigma_{k, I}^{2}= & \Re\left[\mathbf{w}_{k}^{H} \mathbf{H}\right] \underline{\mathbf{V}}_{k, I} \Re\left[\mathbf{H}^{H} \mathbf{w}_{k}\right] \\
& -\Im\left[\mathbf{w}_{k}^{H} \mathbf{H}\right] \underline{\mathbf{V}}_{k, Q} \Im\left[\mathbf{H}^{H} \mathbf{w}_{k}\right]+\sigma_{n}^{2} \mathbf{w}_{k}^{H} \mathbf{w}_{k} \\
\sigma_{k, Q}^{2}= & \Re\left[\mathbf{w}_{k}^{H} \mathbf{H}\right] \underline{\mathbf{V}}_{k, Q} \Re\left[\mathbf{H}^{H} \mathbf{w}_{k}\right] \\
& -\Im\left[\mathbf{w}_{k}^{H} \mathbf{H}\right] \underline{\mathbf{V}}_{k, I} \Im\left[\mathbf{H}^{H} \mathbf{w}_{k}\right]+\sigma_{n}^{2} \mathbf{w}_{k}^{H} \mathbf{w}_{k}
\end{aligned}
$$

where $\underline{\mathbf{V}}_{k, I}=\operatorname{diag}\left[v_{1, I} \ldots v_{k-1, I} \quad 0 \quad v_{k+1, I} \ldots v_{K, I}\right]$, $\underline{\mathbf{V}}_{k, Q}=\operatorname{diag}\left[v_{1, Q} \ldots v_{k-1, Q} 0 v_{k+1, Q} \ldots v_{K, Q}\right]$, with $v_{k^{\prime}, I}=$ $\mathrm{E}\left[\Re^{2}\left[s_{k^{\prime}}\right]\right]-\Re^{2}\left[\bar{s}_{k^{\prime}}\right]$ and $v_{k^{\prime}, Q}=\mathrm{E}\left[\Im^{2}\left[s_{k^{\prime}}\right]\right]-\Im^{2}\left[\bar{s}_{k^{\prime}}\right]$. Using the Gaussian distribution assumption, the extrinsic information delivered by the MSER MUD can be expressed as $L_{E}\left(b_{k}(i)\right)=$

$$
\begin{aligned}
& \sum_{\forall s^{(m, n)}:} \exp \left(\frac{-\left(\Re\left[\hat{s}_{k}\right]-\mu_{k, I}^{(m, n)}\right)^{2}}{2 \sigma_{k, I}^{2}}\right) \prod_{\forall i^{\prime} \neq i} \mathrm{P}\left(b_{k}^{(m, n)}\left(i^{\prime}\right)\right) \\
& \ln \frac{b^{(m, n)}(i)=0}{} \\
& \sum_{\substack{\forall s^{(m, n):} \\
(m, n)(i)=1}} \exp \left(\frac{-\left(\Re\left[\hat{s}_{k}\right]-\mu_{k, I}^{(m, n)}\right)^{2}}{2 \sigma_{k, I}^{2}}\right) \prod_{\forall i^{\prime} \neq i} \mathrm{P}\left(b_{k}^{(m, n)}\left(i^{\prime}\right)\right)
\end{aligned}
$$

when $b_{k}(i)$ is mapped to the real part of $s_{k}$, and $L_{E}\left(b_{k}(i)\right)=$

$$
\begin{aligned}
& \sum_{\forall s^{(m, n)}:} \exp \left(\frac{-\left(\Im\left[\hat{s}_{k}\right]-\mu_{k, Q}^{(m, n)}\right)^{2}}{2 \sigma_{k, Q}^{2}}\right) \prod_{\forall i^{\prime} \neq i} \mathrm{P}\left(b_{k}^{(m, n)}\left(i^{\prime}\right)\right) \\
& \ln \frac{b^{(m, n)}(i)=0}{=0} \\
& \sum_{\forall s^{(m, n)}:} \exp \left(\frac{-\left(\Im\left[\hat{s}_{k}\right]-\mu_{k, Q}^{(m, n)}\right)^{2}}{2 \sigma_{k, Q}^{2}}\right) \prod_{\forall i^{\prime} \neq i} \mathrm{P}\left(b_{k}^{(m, n)}\left(i^{\prime}\right)\right) \\
& b^{(m, n)}(i)=1
\end{aligned}
$$




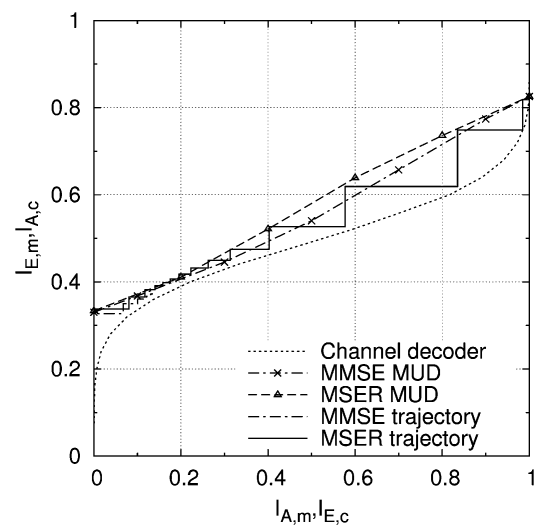

Fig. 3. EXIT charts and simulated trajectories of the iterative MMSE and MSER receivers supporting $K=316 \mathrm{QAM}$ users at $E_{b} / N_{0}=7.5 \mathrm{~dB}$.

when $b_{k}(i)$ is mapped to the imaginary part of $s_{k}$, where $b^{(m, n)}(i)$ denotes the $i$ th bit of $s^{(m, n)}$ and $\mathrm{P}\left(b_{k}^{(m, n)}\left(i^{\prime}\right)\right)=$ $(1 / 2)\left(1+\operatorname{sgn}\left((1 / 2)-b^{(m, n)}\left(i^{\prime}\right)\right) \cdot \tanh \left(L_{A}\left(b_{k}\left(i^{\prime}\right)\right) / 2\right)\right)$.

\section{EXIT CHART ANALYSIS}

The EXIT chart analysis computes the MI between the LLRs and the corresponding bits [11]. An EXIT chart is shown in Fig. 3 for the simulated system investigated in Section V. Let $I_{A}$ denote the MI between the a priori values $L_{A}$ and the corresponding bit-sequence, while $I_{E}$ denote the MI between the extrinsic values $L_{E}$ and the corresponding bit-sequence. The EXIT function of the channel decoder is defined by $I_{E, c}=$ $f_{c}\left(I_{A, c}\right)$, which maps the input variable $I_{A, c}$ to the output variable $I_{E, c}$, and the value of $I_{E, c}$ in the range $[0,1]$ characterizes the quality of the output LLRs of the decoder components. In the multiuser scenario, the MUD's EXIT curve recorded for the desired user depends on all the other $(K-1)$ users' channel decoder output MI. In our simulations, all the users' signal-tonoise ratios (SNRs) are identical. Hence, the turbo MUD can average all the users' MIs in order to simplify the EXIT chart function to $I_{E, m}=f_{m}\left(I_{A, m}, E_{b} / N_{0}\right)$.

The output of one of the two constituent components is the input of the other; hence, both transfer functions are shown in the same EXIT plane having coordinate axes of $\left(I_{A, m}=I_{E, c}\right)$, $\left(I_{E, m}=I_{A, c}\right)$. The staircase-shaped lines in Fig. 3, connecting the MI points evaluated during each iteration, are referred to as the detection trajectory. An infinitesimally low BER may be attained, when there is a so-called open tunnel between the EXIT curves of the decoder and the MUD. This graphical representation gives us an immediate insight into the number of detection iterations required for attaining the best possible BER performance.

\section{Simulation Results}

The system employs a two-element antenna array to support $K=3$ 16QAM users. All the users have the same transmit power. Each user employs a different randomly generated interleaver. The interleaver length of each user is $2 \times 10^{4}$ bits. All the users have the same channel coefficients of $h_{k}=1.0+j 0.0$, $1 \leq k \leq 3$, and employ the same rate-1/2 and constraint-length 4 non-systematic convolutional code using the octally represented generators $(15,17)$. The arrival angles of the users' signals are $68^{\circ}, 15^{\circ}$, and $-24^{\circ}$, respectively.

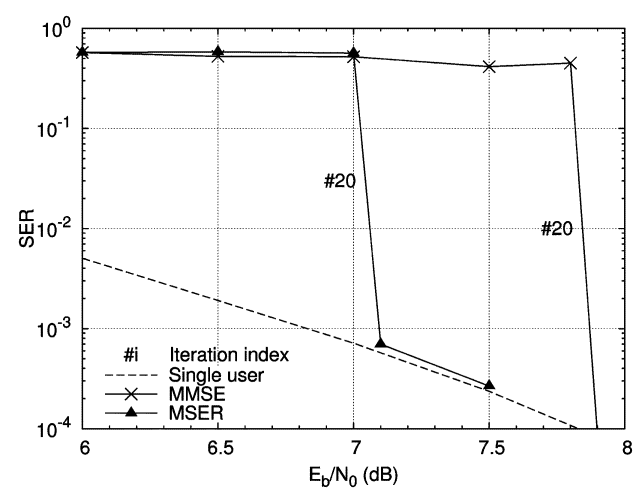

Fig. 4. SER comparison of the MMSE and MSER iterative beamforming receivers for the 16QAM system supporting $K=3$ users.

Fig. 3 shows the EXIT curves and the simulated trajectories of the iterative MMSE and MSER 16QAM beamforming receivers at $E_{b} / N_{0}=7.5 \mathrm{~dB}$. For this system, the MMSE and MSER MUDs have almost the same output $I_{E, m}$ value at both the axes at $I_{A, m}=0$ and $I_{A, m}=1$. Between these two points of intersection, the MMSE MUD has the lower EXIT curve, and the MSER's EXIT curve reaches a higher $I_{E, m}$ value. Fig. 4 shows the SER versus SNR performance of the MMSE and MSER beamforming receivers, in contrast to the single-user performance. It can be seen that after $i=20$ iterations, both the iterative systems approach the single-user performance. The MSER system has a lower operating SNR threshold, which is $0.8 \mathrm{~dB}$ lower than that of the MMSE system.

\section{REFERENCES}

[1] C. Berrou, A. Glavieux, and P. Thitimajshima, "Near Shannon limit error correcting coding and decoding: Turbo codes," in Proc. ICC 1993, Geneva, Switzerland, May 1993, vol. 2, pp. 1064-1070.

[2] S. Benedetto, D. Divsalar, G. Montorsi, and F. Pollara, "Serial concatenation of interleaved codes: Performance analysis, design, and iterative decoding," IEEE Trans. Inf. Theory, vol. 44, no. 3, pp. 909-926, May 1998.

[3] C. Douillard, A. Picart, M. Jézéquel, P. Didier, C. Berrou, and A. Glavieux, "Iterative correction of intersymbol interference: Turboequalization," Eur. Trans. Commun., vol. 6, pp. 507-511, Sep.-Oct. 1995.

[4] M. Tüchler, A. C. Singer, and R. Koetter, "Minimum mean squared error equalization using a priori information," IEEE Trans. Signal Process., vol. 50, no. 3, pp. 673-6820, Mar. 2002.

[5] M. Tüchler, R. Koetter, and A. C. Singer, "Turbo equalization: Principles and new results," IEEE Trans. Commun., vol. 50, no. 5, pp. 754-767, May 2002

[6] X. Wang and H. V. Poor, "Iterative (Turbo) soft interference cancellation and decoding for coded CDMA," IEEE Trans. Commun., vol. 47, no. 7, pp. 1046-1060, Jul. 1999.

[7] S. Chen, N. N. Ahmad, and L. Hanzo, "Adaptive minimum bit error rate beamforming," IEEE Trans. Wireless Commun., vol. 4, no. 2, pp. 341-348, Mar. 2005.

[8] S. Chen, L. Hanzo, N. N. Ahmad, and A. Wolfgang, "Adaptive minimum bit error rate beamforming assisted receiver for QPSK wireless communication," Digit. Signal Process., vol. 15, no. 6, pp. 545-567, Mar. 2005.

[9] C.-C. Yeh and J. R. Barry, "Adaptive minimum symbol-error rate equalization for quadrature amplitude modulation," IEEE Trans. Signal Process., vol. 51, no. 12, pp. 3263-3269, Dec. 2003.

[10] S. Chen, H.-Q. Du, and L. Hanzo, "Adaptive minimum symbol error rate beamforming assisted receiver for quadrature amplitude modulation systems," in Proc. VTC 2006-Spring, Melbourne, Australia, May 2006, vol. 5, pp. 2236-2240.

[11] S. ten Brink, "Convergence behavior of iteratively decoded parallel concatenated codes," IEEE Trans. Commun., vol. 40, no. 10, pp. 1727-1737, Oct. 2001.

[12] K. Li and X. Wang, "Exit chart analysis of turbo multiuser detection," IEEE Trans. Wireless Commun., vol. 4, no. 1, pp. 300-311, Jan. 2005. 